Copyright (C) 2014 IEEE. Personal use of this material is permitted. Permission from IEEE must be obtained for all other uses, in any current or future media, including reprinting/republishing this material for advertising or promotional purposes, creating new collective works, for resale or redistribution to servers or lists, or reuse of any copyrighted component of this work in other works. 


\section{Reliable Green Routing Using Two Disjoint Paths}

\author{
Gongqi Lin, Sieteng Soh, Mihai Lazarescu \\ Curtin University of Technology \\ Perth Australia \\ Gongqi.lin@postgrad.curtin.edu.au
}

\author{
Kwan-Wu Chin \\ University of Wollongong \\ Wollongong Australia \\ kwanwu@uow.edu.au
}

\begin{abstract}
Network robustness and throughput can be improved by routing each demand $d$ via two disjoint paths (2DP). However, 2DP routing increases energy usage while providing lower link utilization and redundancy. In this paper, we address an NPcomplete problem, called 2DP-EAR, that aims to switch off redundant nodes and links while guaranteeing two constraints: traffic demands must be afforded 2DP, and maximum link utilization. We design an efficient heuristic, called 2DP by Nodes First (2DP-NF). We have extensively evaluated the performance of 2DP-NF on both real and/or synthetic topologies and traffic demands. As compared to using Shortest Path routing, on the GÉANT network, 2DP-NF can save around $20 \%$ energy by switching off links only with negligible effects on path delays and link utilization, even for MLU below 30\%. Furthermore, 2DP-NF can obtain $39.7 \%$ power savings by switching off both nodes and links on the GÉANT network.
\end{abstract}

Keywords - robustness; thoughput; power savings; maximum link utilization; two disjoint paths

\section{INTRODUCTION}

Recently, disjoint path routing has been proposed to improve network reliability and throughput $[1,2]$. Two paths between source node $s_{d}$ and destination node $t_{d}$ are node (link)disjoint, called 2DP-N (2DP-L), if they have no common nodes (links). In [3], the authors combine link/node-disjoint paths with QoS routing to guarantee various performance requirements, e.g., reliability and delay. While overprovisioning resources to provide backup paths using traffic engineering (TE) can help improve reliability, it exhibits poor energy efficiency, especially at low traffic load periods. Recent reports $[4,5]$ show that power consumption of large wired networks has increased tremendously. In fact, the energy consumption of the Information and Communication Technologies (ICT) sector will grow from $22 \mathrm{GW}$ in 2007 to $95 \mathrm{GW}$ in 2020 , generating $1.4 \mathrm{Gt}$ of $\mathrm{CO} 2$ or approximately $2.8 \%$ of global warming by 2020 [4].

Recently, the authors of [6] propose a solution that minimizes powered-on links by routing demands with $2 \mathrm{DP}-\mathrm{L}$ under maximum link utilization constraint. However, their work only considers switching off links and does not address the 2DP-N problem. This problem is significant because routers consume significantly more power as compared to links [7]. Reference [8] proposes a solution to switch-off both links and nodes. However, their solution [8] does not address 2DP routing, which affects fault-tolerance [9]. We note that references [10] and [11] have proposed power-aware routing algorithms, but they do not require generated paths to be linkdisjoint or node-disjoint.

In this paper, we extend the work in [6] to reduce power by switching off both nodes and links. Specifically, our contributions are twofold. First, we formulate a problem, called Two Disjoint Paths Energy-Aware Routing (2DP-EAR), to minimize the power consumption of network resources by switching off both unused nodes and links subjected to two constraints: (i) link utilization must be no larger than a given $U$, and (ii) there are at least $T$ fractions of routes that use 2DP. Our model aims to reduce energy usage of networks that support 2DP routing to improve fault-tolerance and throughput. The formulation extends that of [6], which only considers switching off links. Second, we propose a novel algorithm, called Two Disjoint Paths by Nodes First (2DP-NF), to solve 2DP-EAR. Our 2DP-NF prioritizes switching off nodes to links since nodes consume an order of magnitude more energy [7]. Our extensive simulation results show the advantage of using 2DPNF. For example, for the GÉANT network, 2DP-NF can obtain $39.7 \%$ power savings.

The rest of the paper is organized as follows. Section II gives an overview of the 2DP-EAR problem and summarize of notations. Section III describes our heuristic algorithm, 2DP$\mathrm{NF}$, to solve the problem. Section IV evaluates the performance of 2DP-NF using both real and/or synthetic topologies and data. Finally, Section V concludes the paper.

\section{PROBLem FORMULATION}

In this section, we describe our problem, Two Disjoint Paths Energy-Aware Routing (2DP-EAR). Without loss of generality, we only define the problem for two link-disjoint paths (2DP-L); note that a two node-disjoint paths (2DP-N) is also 2DP-L.

\section{A. Notation}

Consider a computer network that is represented by a weighted directed graph $G(V, E)$, where $V$ is the set of $n$ nodes, and $E$ is the set of $m$ links. Each node in $V$ represents a router, and each link $(i, j) \in E$ between nodes $v_{i}$ and $v_{j}\left(v_{i}, v_{j} \in V, v_{i} \neq v_{j}\right)$ represents a communication channel with capacity/bandwidth $c_{i j}>0$. For edge $(i, j) \in E$, let $0 \leq f_{i j} \leq c_{i j}$ be the traffic load through edge $(i, j)$, and hence, its utilization is given by $u_{i j}=f_{i j} / c_{i j}$. The utilization $u_{i j}$ is bounded by a threshold $0 \leq U \leq 1.0$ and is set by network operators; i.e., $u_{i j} \leq U$. A link's remaining capacity is defined as $r_{i j}=U^{*} c_{i j} f_{i j} \geq 0$. We assume each link $(i, j) \in E$ can be 
switched-off independently. Each node $v$ and link $(i, j)$ consumes equal power $p_{v}$ and $p_{i j}$ respectively. Let $E_{v}$ be the set of links connected to a node $v$, and $f_{v}=\sum_{(v, j) \in E_{v}} f_{v j}$ and $c_{v}=\sum_{(v, j) \in E_{v}} c_{v j}$ represent the total throughput rate and total throughput capacity of node $v$ respectively.

Let $D$ be a set of all demands in $G(V, E)$, and $\left(s_{d}, t_{d}, b_{d}\right)$ denote a traffic demand $d=1,2, \ldots,|D|$ between source node $s_{d} \in V$ and terminal node $t_{d} \in V$, where $b_{d}$ is the amount of traffic exchanged between these nodes. For each demand $d$, let $S P_{d}=\left\{s p_{d q} \mid\right.$ all paths for demand $d$ indexed by an integer number $q>0\}$. Let $\mathrm{B}\left(s p_{d q}\right)$ be the capacity of any path $s p_{d q} \in S P_{d}$ calculated by taking the smallest $r_{i j}$ for each link $(i, j) \in s p_{d q}$. Two $\left(s_{d}, t_{d}\right)$ paths are link-disjoint if they have no common links. Let $D P_{d}=\left\{d p_{d l} \mid\right.$ all two link-disjoint paths for demand $d$ indexed by an integer number $l>0\}$. Note that $d p_{d l}=\left\{s p_{d x}, s p_{d y}\right\}$, where $s p_{d x}, s p_{d y} \in S P_{d}$ have no links in common. We use $R_{d}$ to denote a route for demand $d$ that contains a single path $s p_{d q}$, a 2DP-L $d p_{d l}$ or any multiple $\left(s_{d}, t_{d}\right)$ paths with sufficient capacity to route $b_{d}$.

Let $R^{\beta}$ be a route set $\beta$ that contains all $R_{d}$ for each demand $d \in D$, i.e., $R^{\beta}=\left\{R_{d}^{\beta} \mid R_{d}^{\beta} \subseteq S P_{d}\right.$ and/or $\left.R_{d}^{\beta} \in D P_{d}\right\}$. The set of all possible solutions to route demands in $D$ is denoted as $R=\left\{R^{\beta} \mid\right.$ $\beta=1,2, \ldots,|R|\}$. We use $T P^{\beta} \subseteq R^{\beta}$ to represent a set of all $R_{d}^{\beta}$ $\in R^{\beta}$ that includes at least one $d p_{d l} \in D P_{d}$, and $\mathrm{M}\left(R^{\beta}\right)$ be the fraction of $\left(s_{d}, t_{d}\right)$ pairs in $R^{\beta}$ that are routed over 2DP-L, i.e., $\mathrm{M}\left(R^{\beta}\right)=\left|T P^{\beta}\right| /\left|R^{\beta}\right|$; we set $T_{\max }=\max \left\{\mathrm{M}\left(R^{\beta}\right)\right\}$. Finally, let $\mathrm{S}\left(R^{\beta}\right)$ be the total power of active nodes and links used in set $R^{\beta}$ and $\mathrm{U}\left(R^{\beta}\right)=\max \left\{f_{i j} / c_{i j} \mid \forall(i, j) \in R^{\beta}\right\}$.

\section{B. Problem Statement}

Consider a tuple $(G, D, T, U)$, where $G(V, E)$ is a network topology, $D$ is a set of traffic demands, $0 \leq T \leq T_{\max }$ is the required fraction of the total number of routes that contains at least one 2DP-L, and $U$ is a configured threshold of maximum link utilization. We define the 2DP-EAR problem as follows.

2DP-EAR: Find a set $R_{\min } \in R$ that can be used to route all demands in $D$ such that

$$
\begin{aligned}
& \mathrm{S}\left(R_{\text {min }}\right)=\min \left\{p_{v} \mathrm{~V}\left(R^{\beta}\right)+p_{i j} \mathrm{E}\left(R^{\beta}\right) \mid R^{\beta} \in R\right\} \\
& \mathrm{M}\left(R_{\text {min }}\right) \geq T \\
& \mathrm{U}\left(R_{\text {min }}\right) \leq U
\end{aligned}
$$

Note that $\mathrm{V}\left(R^{\beta}\right)$ and $\mathrm{E}\left(R^{\beta}\right)$ are the number of power-on nodes and links in each $R^{\beta} \in R$ respectively. The term $\mathrm{S}\left(R_{\min }\right)$ represents the power consumption of all nodes and links in $R_{\min }$ subject to constraints (2) and (3). Constraint (2) requires there to be at least $T$ fraction of routes in $R_{\min }$ that include at least one 2DP-L, while constraint (3) ensures the link utilization of each link used in $R_{\min }$ be no larger than $U$. The 2DP-EAR problem is a variant of the multi-constrained path (MCP) problem since it aims to generate an optimal set of feasible routes $R_{\min }$ subject to two constraints, i.e., (2) and (3). Since MCP with more than one constraint is known to be NP-complete [12], we conclude that 2DP-EAR is NP-complete.

\section{GREEN ROUTING ALGORITHMS}

In this section, we present our approach, Two Disjoint Paths by Node First (2DP-NF), shown in Fig. 1, to heuristically solve the 2DP-EAR problem. Initially, the set of remaining nodes $V_{r}$ and links $E_{r}$ are $V$ and $E$; 2DP-NF produces $V-V_{r}$ and $E-E_{r}$ as its outputs. We first describe 2DP-NF for 2DP-L, called 2DP-NF-L. We show how to use 2DP-NF for 2DP-N, called 2DP-NF-N, in Section III.B, and discuss the time complexity of 2DP-NF in Section III.C.

\section{A. 2DP-NF-L Algorithm}

As shown in Fig. 1, 2DP-NF-L has six main steps. Step 1 uses Yen's algorithm [13] to generate $k \geq 1$ shortest paths, $K S P_{d}=\left\{s p_{d 1}, s p_{d 2}, \ldots, s p_{d k}\right\}$, for each demand $d$; we assume the delay of each link to be one unit, and thus each path length can measured by its hop count. Note that for each demand $d$, we have $K S P_{d} \subseteq S P_{d}$. Let $K S P=\left\{K S P_{d}|d=1, \ldots| D \mid,\right\}$ be the set of all $k$-shortest paths for all demands $D$.

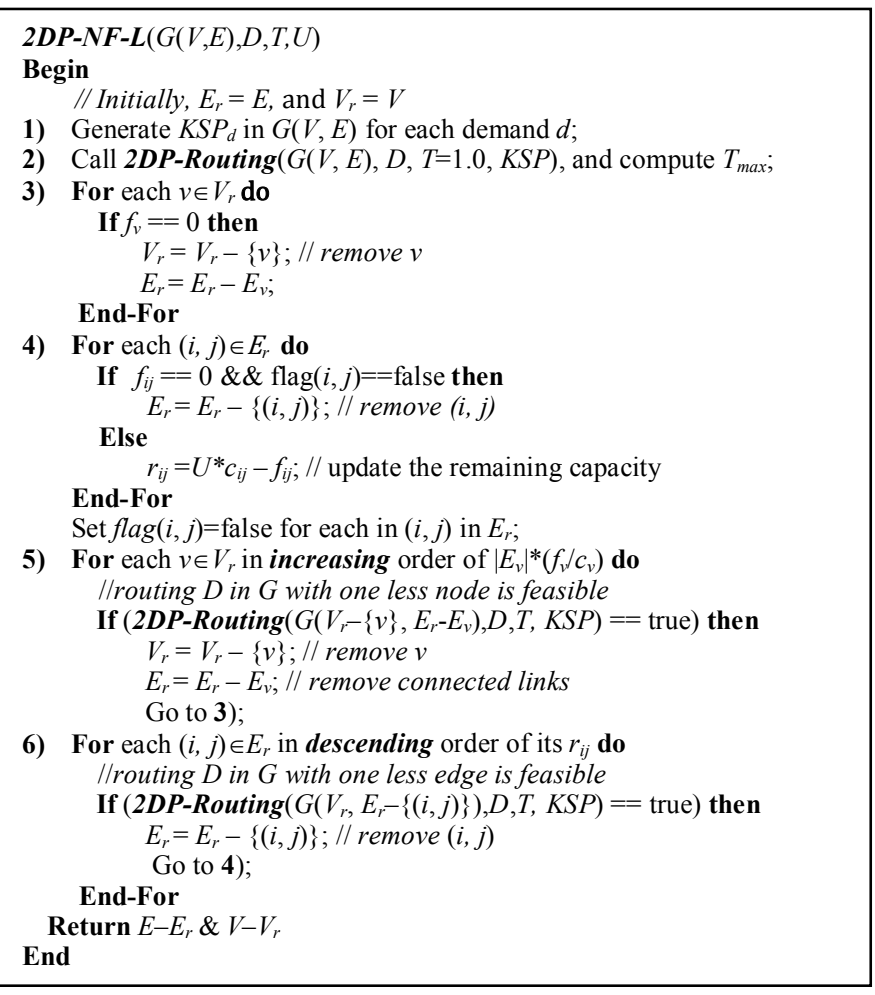

Figure 1. 2DP-NF-L Algorithm

Step 2 uses function 2DP-Routing() to distribute the traffic of each demand $d \in D$ through its candidate paths $K S P_{d}$, and computes $T_{\max }$; we will describe 2DP-Routing() and $T_{\max }$ 's calculation later in this section. Step 3 switches off each unused node $v$, i.e., each node with $f_{v}=0$, and its incident links. Step 4 turns off each unused link $(i, j)$ with variable $f \operatorname{lag}(i$, $j)=$ false, i.e., each link with $f_{i j}=0$, and calculates the spare capacity $r_{i j}=U^{*} c_{i j}-f_{i j}$ of other links. Specifically, we set $f \operatorname{lag}(i$, $j)=$ true for each link $(i, j)$ in a 2DP so that the links are not switched off, i.e., the path comprises of the links becomes a backup path. Step 5 aims to switch-off node $v$, starting from $v$ with fewest connected links $\left|E_{v}\right|$ and lowest link utilization $\left(f_{v} / c_{v}\right)$; intuitively, because such node is used by fewer flows, rerouting these flows successfully is more probable. If there 
exists a feasible $R^{\beta}$ without using $v$ and its incident links $E_{v}$, i.e., 2DP-Routing() returns true, the step switches off node $v$ and all links in $E_{v}$ and repeats Step 3 to update affected nodes and links; otherwise, Step $\mathbf{5}$ is repeated using the next candidate node. Step 6 aims to switch off each link with the largest spare capacity; this step uses 2DP-Routing() to check if all traffic can be routed through the remaining links in $E_{r}$, while satisfying the required constraints. The step is repeated for the next candidate link if 2DP-Routing() fails to generate a feasible $R^{\beta}$; otherwise, we repeat Step 4 .

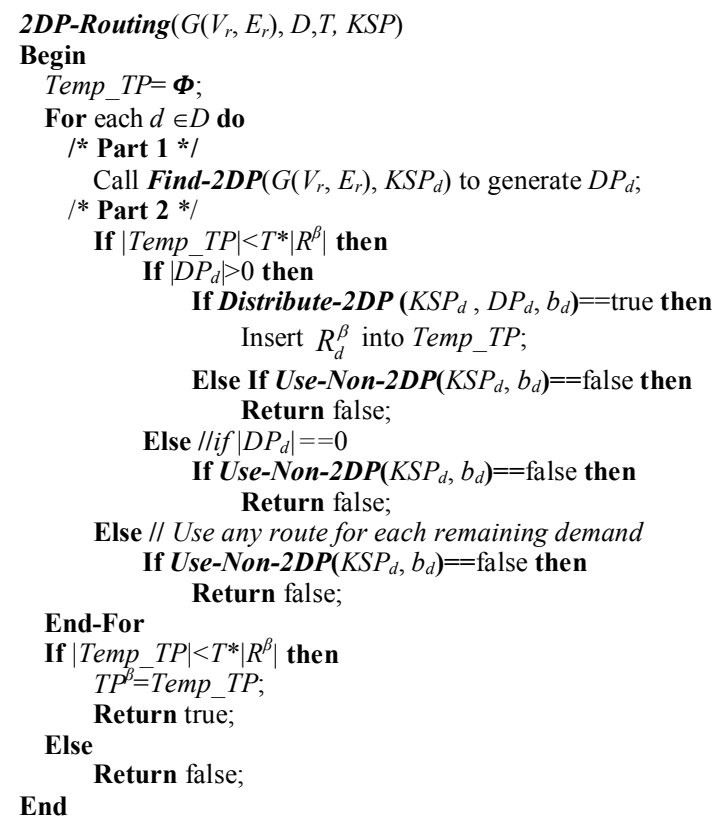

Figure 2. Function 2DP-Routing()

Function 2DP-Routing(), shown in Fig. 2, contains two parts. Part 1 uses function Find-2DP(), shown in Fig. 3, to generate each 2DP-L, $d p_{d l}=\left\{\left(s p_{d x}, s p_{d y}\right) \mid s p_{d x} \in K S P_{d}\right\}$, for each demand $d$. Specifically, function Find-2DP() generates a graph $G_{1}\left(V_{r}, E_{1}\right)$ by deleting all links in each $s p_{d x} \in K S P_{d}$ from $G$. Then, it uses Yen's algorithm to generate $k$-shortest paths from $G_{1}\left(V_{r}, E_{1}\right)$, and stores the paths in the set $K S P_{d}^{\prime}$. Finally, it generates $d p_{d l}=\left\{s p_{d x}, s p_{d y}\right\}$ for each path $s p_{d y} \in K S P^{\prime}{ }_{d}$ that has no common links with $s p_{d x}$, and stores the pair in set $D P_{d}$ in increasing path length $\max \left\{L\left(s p_{d x}\right), L\left(s p_{d y}\right)\right\}$ order.

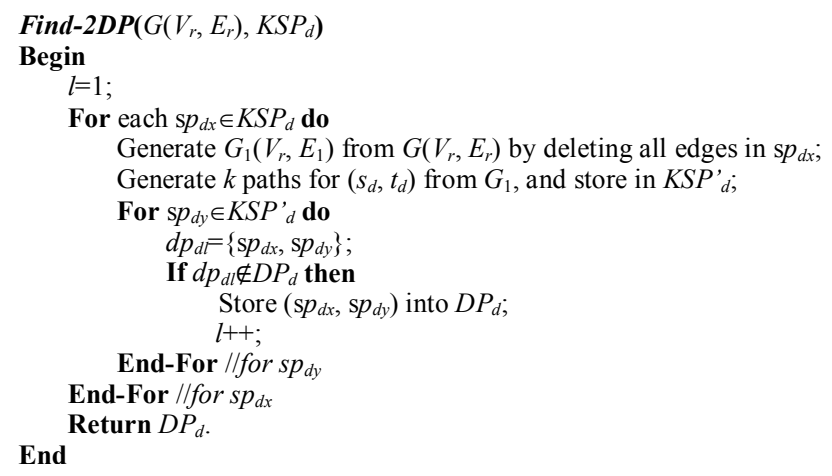

Figure 3. Function Find-2DP()
Part 2 routes all traffic demands subject to constraint $T$. It uses the set Temp_TP, initially empty, to store $R_{d}^{\beta}$ for each demand $d$. If $\mid$ Temp_TP $\left|\geq T^{*}\right| R^{\beta} \mid$ is true, the remaining traffic demands can be routed via any routes using function UseNon-2DP( $)$, shown in Fig. 4. The function aims to distribute each demand $\left(s_{d}, t_{d}, b_{d}\right)$ via its shortest path. However, if the path does not have sufficient capacity, the function will route remaining flow through the next available shortest path. The step is repeated until $b_{d}$ is completely routed and the function returns true; otherwise, it returns false and 2DP-Routing() returns false since it fails to route all demands in $D$. Note that Use-Non-2DP () considers shortest paths and therefore uses fewer links for routing a demand than using 2DP-L.

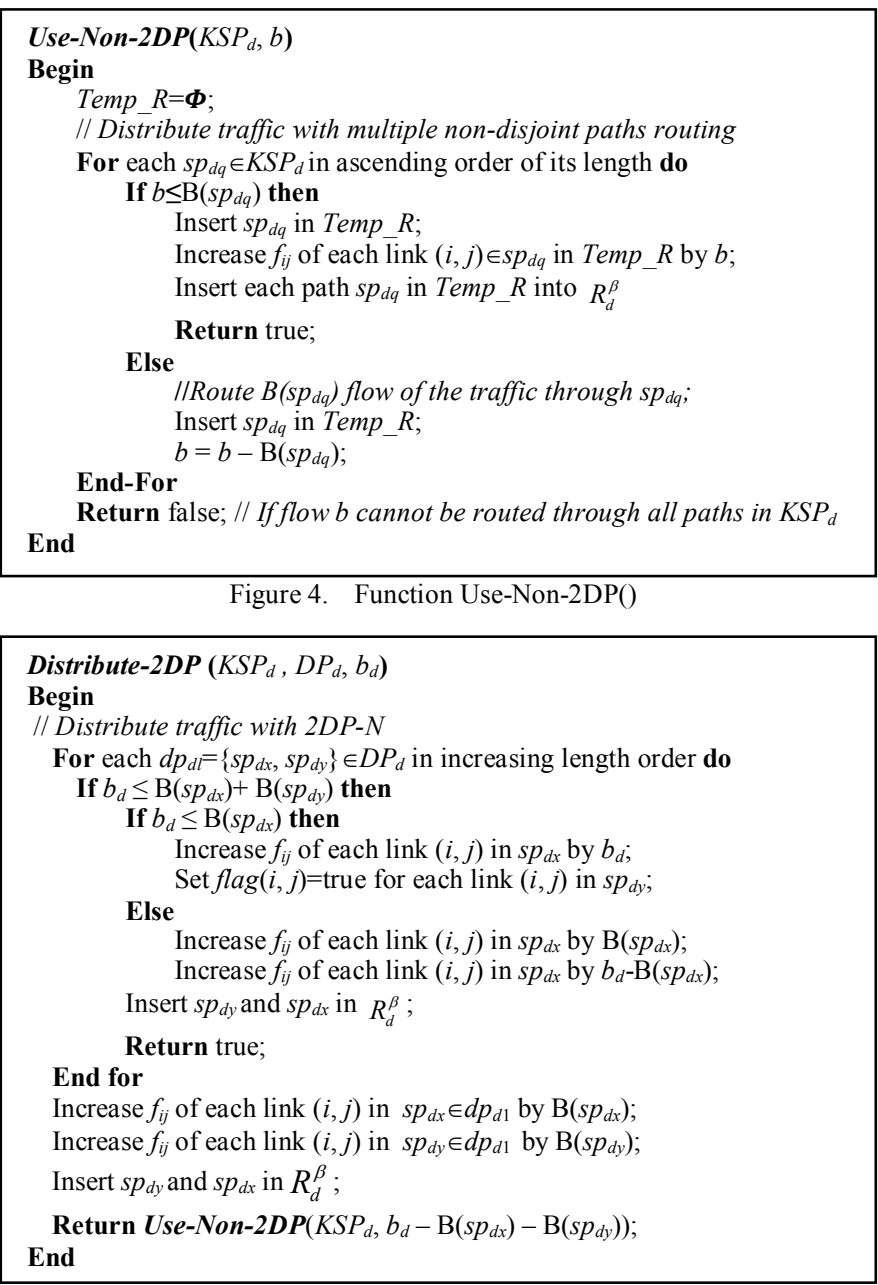

Figure 5. Function Distribute_2DP()

However, if $\mid$ Temp_TP $\left|<T^{*}\right| R^{\beta} \mid$ and at least one 2DP-L of demand $d$ exists, i.e., $\left|D P_{d}\right|>0$, Part 2 uses Distribute-2DP( $)$, described later, to distribute traffic of demand $d$ via its $d p_{d l}$ and insert $R_{d}^{\beta}$ into Temp_TP. For each demand $d$, if $\left|D P_{d}\right|=0$ or Distribute-2DP() returns false, 2DP-Routing() uses Use-Non2DP( ) to route $b_{d}$ via one or more paths starting from the shortest path in $K S P_{d}$. Finally, if all traffic demands are allocated successfully and the requirement $\left|T e m p \_T P\right| \geq T^{*}\left|R^{\beta}\right|$ is satisfied, Temp_TP=TP $P^{\beta}$ and 2DP-Routing () returns true. When function 2DP-Routing() returns true, i.e., it has 
successfully routed all demands in $D$, it updates $R_{d}^{\beta}$ for each demand $d$ and the total flows on each link $(i, j) \in E_{r}$, i.e., $f_{i j}$. When the function returns false, it will maintain the previous routing $R^{\beta-1}$.

For each demand with $\left|D P_{d}\right|>0$, Distribute-2DP(), shown in Fig. 5, routes traffic demand $b_{d}$. The function aims to route the traffic $d$ through its 2DP-L, i.e., $\left(s p_{d x}, s p_{d y}\right) \in d p_{d l}$, and we assume $\mathrm{B}\left(s p_{d x}\right) \leq \mathrm{B}\left(s p_{d y}\right)$. If $b_{d} \leq \mathrm{B}\left(s p_{d x}\right)$ then it routes $b_{d}$ through only a single path $s p_{d x}$, and sets $f \operatorname{lag}(i, j)=$ true for each $(i, j)$ backup path $s p_{d y}$ that can enhance routing reliability. This flow distribution is different than in [10] that splits flow equally. If $\mathrm{B}\left(s p_{d x}\right) \leq b_{d} \leq \mathrm{B}\left(s p_{d x}\right)+\mathrm{B}\left(s p_{d y}\right)$ then it routes the traffic volume $\mathrm{B}\left(s p_{d x}\right)$ via $s p_{d x}$, and routes volume $b_{d}-\mathrm{B}\left(s p_{d x}\right)$ via $s p_{d y}$. Note that, $\mathrm{B}\left(s p_{d q}\right)=\min \left\{r_{i j} \mid(i, j) \in s p_{d q}\right\}$. However, if $\mathrm{B}\left(s p_{d x}\right)+$ $\mathrm{B}\left(s p_{d y}\right)<b_{d}$, it uses function Use-Non-2DP( $)$ to distribute the remaining flow, i.e., $b_{d}-\left(\mathrm{B}\left(s p_{d y}\right)+\mathrm{B}\left(s p_{d x}\right)\right)$. Note that Distribute-2DP( $)$ returns false when Use-Non-2DP( $)$ returns false, i.e., it fails to route the remaining flows through the paths in $K S P_{d}$; for this case 2DP-Routing() will use function UseNon-2DP( $)$ to route $b_{d}$. Recall that Step 2 of 2DP-NF-L in Fig. 1 uses function 2DP-Routing() to initialize the traffic distribution in the network. In this step, we set $T=1.0$ so that the function routes each demand through its 2DP-L whenever possible, and thus $T_{\max }$ is set $\left|T P^{1}\right| /|D|$, where $T P^{1}$ includes all demands in $D$ that are routed via 2DP-L in original network.

\section{B. $2 D P-N F-N$}

In general, a network contains fewer 2DP-Ns than 2DP-Ls since each $2 \mathrm{DP}-\mathrm{N}$ is also a 2DP-L, but not vice versa; thus using the latter for routing is more popular [14]. Further, using link-disjoint paths is much more energy efficient than nodedisjoint paths [15]. However, 2DP-N is more resilient to failures than 2DP-L because they protect against both node and link failures. One can use 2DP-NF for applications that require $2 \mathrm{DP}-\mathrm{N}$ by considering only each $2 \mathrm{DP}-\mathrm{L}, d p_{d l}=\left\{s p_{d x}\right.$, $\left.s p_{d y}\right\}$, since each set of $2 \mathrm{DP}-\mathrm{N}$ for each $\left(s_{d}, t_{d}\right)$ is a subset of its set of 2DP-L for each $\left(s_{d}, t_{d}\right)$. However, 2DP-NF-N is expected to switch off less number of links than 2DP-NF-L due to fewer candidate 2DP-N.

\section{Time Complexity of $2 \mathrm{DP}-\mathrm{NF}$}

For 2DP-NF, Yen's algorithm, see Step 1, incurs $O(k n(m+n \log n))$ time. Note that $n$ and $m$ are the total number of nodes and edges in $G$ respectively. In Step 2, 2DPRouting() takes $O\left(|D|\left(k^{2}+m k^{2}\right)\right)=O\left(k^{2} m n^{2}\right)$ time because $|D| \leq n^{2} ;|D|$ is the total number of traffic demands. Step 3 and 4 require searching all nodes and links in $G$ and therefore has a time complexity of $O(n+m)$. Step 5 needs up to $O(n)$ times to check whether a candidate node and its incident links can be deleted. Step 6 takes $O(m)$ to select each candidate. Thus, for $m$ links, this step has complexity $O\left(\mathrm{~m}^{2}\right)$. Since 2DP-Routing() incurs a bound of $O\left(k^{2} m n^{2}\right)$ and called $(n+m)$ times in Step 5 and 6 , the total complexity of $2 D P-N F$ is $O(k n(m+n l o g n)+$ $\left.k^{2} m n^{2}+k^{2} m n^{2}(n+m)\right)=\mathrm{O}\left(k^{2} m^{2} n^{2}\right)$, since $m \leq n^{2}$.

\section{Evaluation}

In this section, we provide detailed experimental findings and present numerical results on the effectiveness of 2DP-NF in reducing power in real networks and its impact on network delay and link utilization. We set $T=T_{\max }$ so that each demand is routed via its 2DP if possible, and use Shortest Path (SP) routing as a benchmark.

\section{A. Experiment Setup}

Table I shows three real topologies, i.e., Abilene [16], GÉANT [17], and Sprint [18], and three synthetic topologies R_Abilene, R_GÉANT and R_Sprint that are used to evaluate the performance of $2 \mathrm{DP}-\mathrm{N} \overline{\mathrm{F}}$ against SP. Each synthetic topology is generated by randomly selecting some nodes from its respective real topology as transit nodes.

TABLE I. NETWORK TOPOLOGIES

\begin{tabular}{|c|c|c|c|}
\hline Network & Access Nodes & Transit Nodes & $\boldsymbol{T}_{\boldsymbol{m a x}}(\mathbf{\%})$ \\
\hline Abilene & 12 & 0 & 83.3 \\
\hline R_Abilene & 10 & 2 & 100 \\
\hline GÉANT & 23 & 0 & 100 \\
\hline R_GÉANT & 10 & 13 & 100 \\
\hline Sprint & 52 & 0 & 37.4 \\
\hline R_Sprint & 26 & 26 & 48.53 \\
\hline
\end{tabular}

From the authors of [16], we obtained 288 traffic matrices for Abilene topology measured on Sep. $5^{\text {th }}, 2004$ for every 5 minutes within 24 hours. For GÉANT, the traffic matrices used were collected on May 5th, 2005 at an interval of 15 minutes; we obtained both the topology and 96 traffic matrices from the authors of [17]. For Sprint, we set its link capacity using the method in [19], and randomly generate a traffic matrix using the gravity model as [11], which is then scaled to obtain 10 different traffic loads. We refer each traffic matrix as TM_X, where $\mathrm{X}$ is the MLU of the network incurred by the traffic when using SP routing; e.g., TM_40 is traffic matrix that produces $\mathrm{MLU}=40 \%$ in Sprint when using SP to route the traffic. For each network, we used Step 2 of both 2DP-NF-L and 2DP-NF-N to obtain $T_{\max }=83.3 \%, \quad T_{\max }=100 \%$, and $T_{\max }=37.4 \%$ for Abilene, GÉANT, and Sprint, respectively.

We consider two types of nodes: access and transit [8]. Access nodes are sources and destinations of information and thus cannot be switched-off. In contrast, transit nodes are neither sources nor destinations of traffic; we aim to switch off as many of these nodes as possible. We assume that OC192/STM-64 (10Gbps) line card is used for all links and thus the maximum delay of a single hop is around $200 \mathrm{~ns}$ [20]. Similar to [8], we assume each cable in link $(i, j)$ has the same power consumption $p_{i j}=0.6 \mathrm{kw}$ and each node $v$ consumes the same power $p_{v}=3 \mathrm{kw}$. The power saving (PS) of each network is calculated as follows,

$$
P S=\left(1-\frac{\left(m-\left|E_{r}\right|\right) p_{i j}+\left(n-\left|V_{r}\right|\right) p_{v}}{m p_{i j}+n p_{v}}\right) \times 100 \%
$$

Our simulations were performed on a Linux PC with 3.07 $\mathrm{GHz}$ CPU and 8 GB RAM.

\section{B. Power off links only}

We aim to see the effect on energy usage by only switching-off links on both 2DP-L and 2DP-N. In this simulation, we use Abilene, GEANT, and Sprint and use the traffic demands described in Section IV.A. Note that each traffic matrix considers all possible traffic demands, i.e., each 
node is the traffic source/sink, and thus no node can be switched off. Hence, we calculate power savings using Equation (4) with $p_{v}=0$. Fig. 6 shows the power saving for the Abilene network using 2DP-NF with different maximum link utilization $U$; we use $\mathrm{N}$ and $\mathrm{L}$ to represent $U$ for 2DP-NF-N and 2DP-NF-L respectively. Note that 2DP-NF-L and 2DPNF-N could not find any links to be switched off at $U \leq 0.2$ and $U \leq 0.3$ respectively. For $U \geq 0.5,2 \mathrm{DP}-\mathrm{NF}-\mathrm{L}$ distributes traffic flow through the same set of paths while 2DP-NF-N requires $U \geq 0.6$ to obtain the same power savings, switching off $20 \%$ links, due to fewer candidate paths for 2DP-NF-N. Fig. 7 presents the power saving of GÉANT using 2DP-NF. Our approach obtains the same power saving for $U=0.3$ to $U=1.0$, and thus we only show the results for $U \geq 0.3$. Notice that $U=0.1$ and $U=0.2$ produce the same power saving, thus we only show $U=0.1$ for $2 \mathrm{DP}-\mathrm{NF}-\mathrm{L}$ and $2 \mathrm{DP}-\mathrm{NF}-\mathrm{N}$. Although the power saving curve of 2DP-NF-L fluctuates during the day due to traffic changes, it always remains around $20.27 \%$ $25.97 \%$. However, as shown in Fig. 7, 2DP-NF-N performs worse than 2DP-NF-L due to fewer candidate paths, especially for $U=0.1$ that produces the worst power saving.
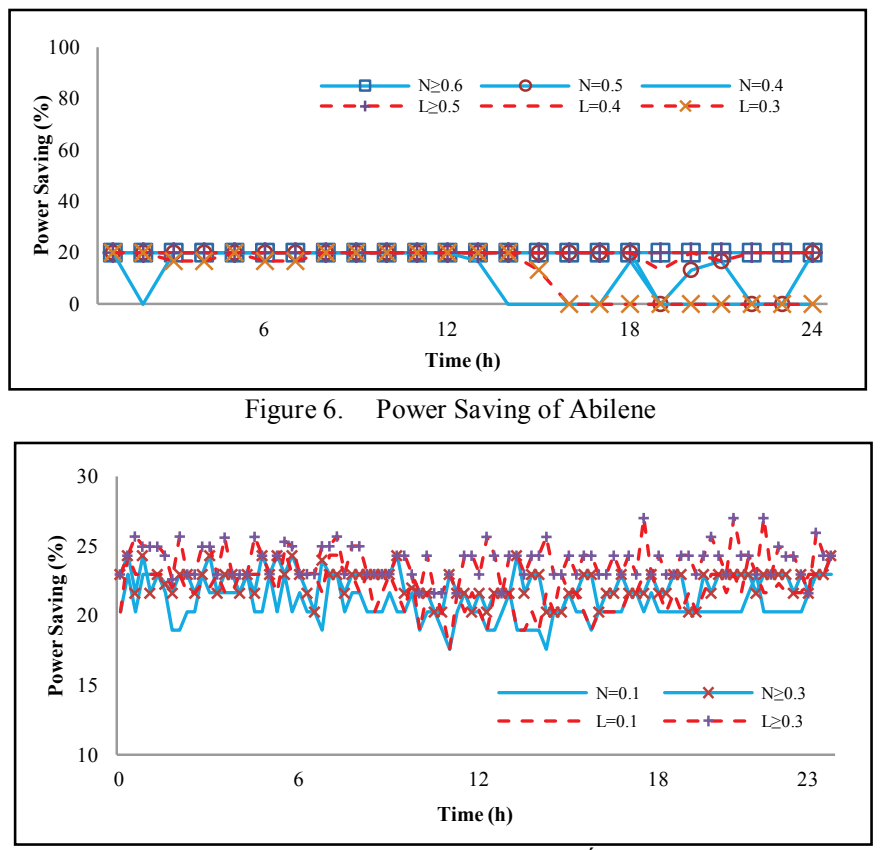

Figure 7. Power Saving of GÉANT

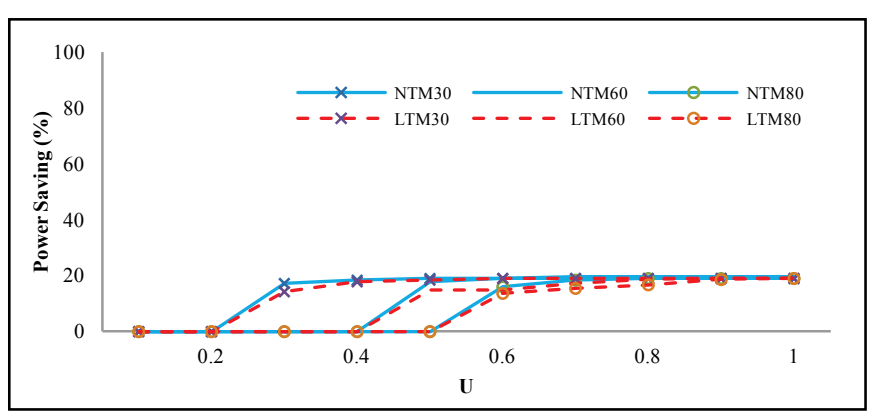

Figure 8. Power Saving of Sprint

Fig. 8 shows the power saving when using 2DP-NF with different TM_X on the Sprint network. We found that 2DP-NF produces the same set of routes with TM_10, TM_20 and TM 30 , and thus we report only the result for TM_30. We found a similar situation for TM 40 to TM 60 , and for TM 70 to TM 80. Note that 2DP-NF failed to save energy for TM -90 and TM_100. Running 2DP-NF-L with TM 30 (labeled LTM30 in the figure) increases PS from $17.26 \%$ to $19.04 \%$ when $U$ is set from 0.3 to 0.5 , and remains at $19.04 \%$ for $U>0.5$. However, 2DP-NF-N with TM 30 (NTM30) saves less power than 2DP-NF-L when $U<0.6$, saving only $14.29 \%$ at $U=0.5$. The reason for this is that with 2DP-L, there are more opportunities for the 2DP-NF-L algorithms to exploit candidate paths. The results for 2DP-NF with TM 60 (LTM60 and NTM60) and TM 80 (LTM80 and NTM80) have the same trend as LTM30. However, 2DP-NF fails to save energy when we set $U<0.5$ for LTM60 and NTM60, and $U<0.6$ for LTM80 and NTM 80.

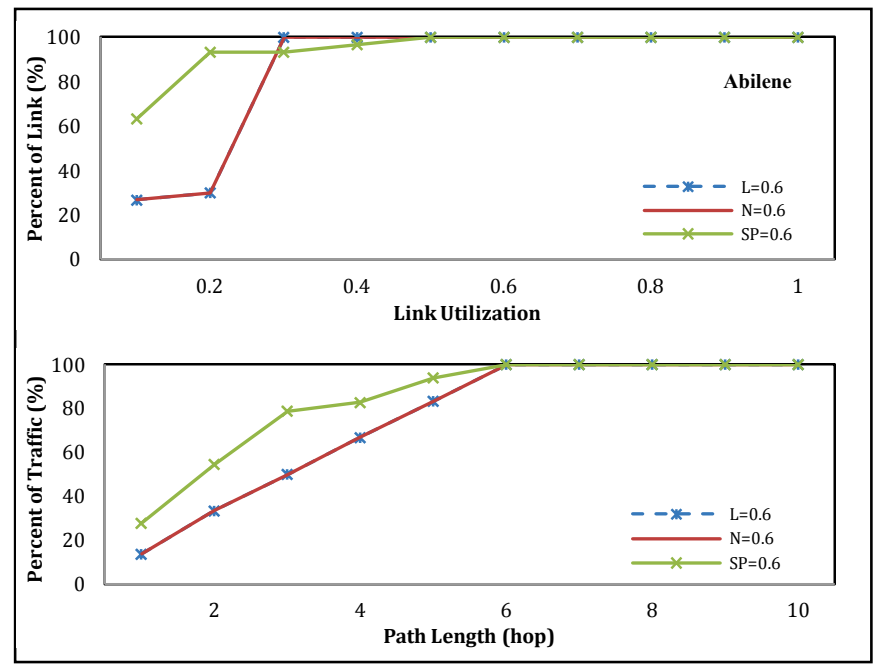

Figure 9. CDF of Link Utilization and Path Length on Abilene

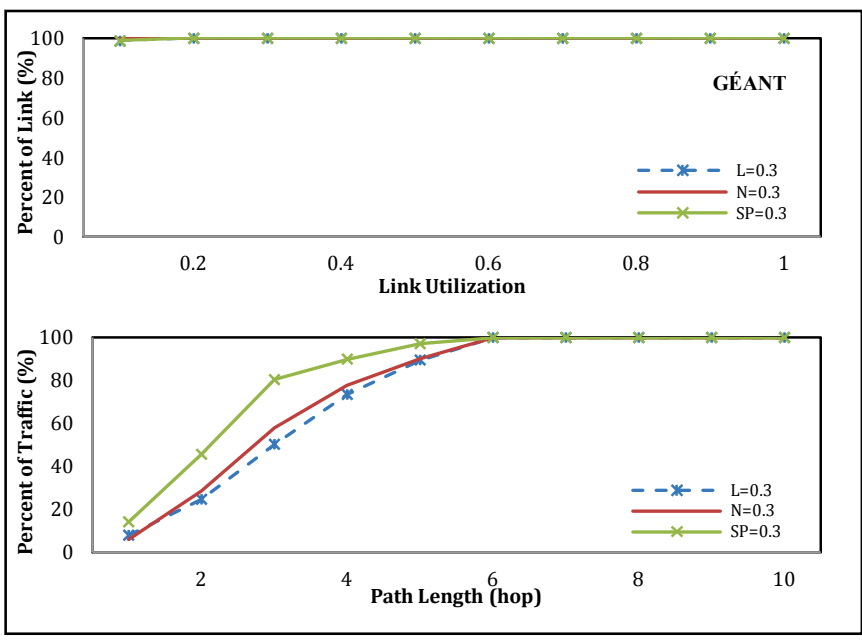

Figure 10. CDF of Link Utilization and Path Length on GÉANT

\section{Effects on Link Utilization and Path Length}

In this subsection, we show the effect of switching off links in Section IV.B on link utilization and path length. For Abilene, shown on the top of Fig. 9, the Cumulative Distribution Function (CDF) of link utilization and path length are the same when we set $U \geq 0.5$. From the figure, we see that 
the results of 2DP-NF-L are the same as 2DP-NF-N at $U=0.6$ due to the use of the same routing paths, which is worse than SP. However, as shown at the bottom of Fig. 9, their path length is no longer than the network diameter (6 hops). For GÉANT, shown in Fig. 10, the utilization of all links is less than 0.1 when we set $U=0.1$ and 0.2 , and thus we do not show results here. When we set $U=0.3$, 2DP-NF-L has $1.35 \%$ links with utilization between 0.1 and 0.2 , which is larger than 2DPNF-N that reaches $100 \%$ links when the link utilization is less than 0.1. In Fig. 10 (bottom), since there are more candidate 2DP-Ls than 2DP-Ns, 2DP-NF-L can switch off more links, i.e., saving more energy, as compared to 2DP-NF-N, and generates more alternative but longer routes. For Sprint, we obtain the CDF of link utilization and path length for TM_30, TM 60 and TM 80. From Fig. 11 (top), we see that 2DP-NF-L performs slightly worse than 2DP-NF-N, with more links having higher link utilization. Similarly, as shown in Fig. 11 (bottom) in term of path length, 2DP-NF-N performs worse than SP, but it obtains better results than 2DP-NF-L. 2DP-NF$\mathrm{N}$ switches-off less number of links as compared to 2DP-NF-L. Notice that for each switched-off link, 2DP-NF needs to generate one or more alternative longer paths, and therefore more powered-off links correlate to longer paths.

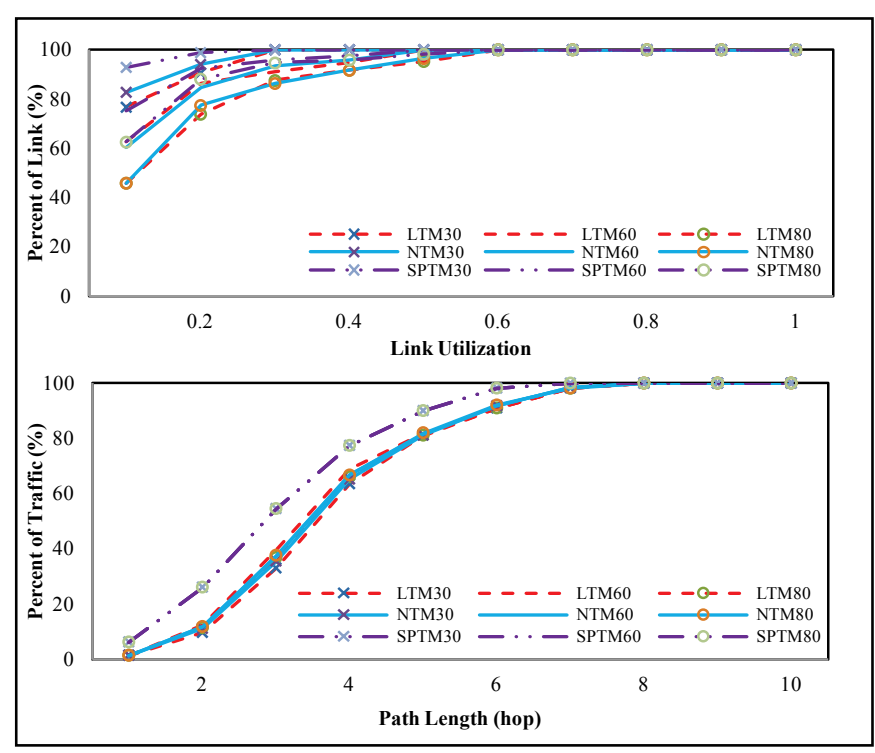

Figure 11. CDF of Link Utilization and Path Length on Sprint

\section{Power off nodes and links}

In this subsection, we use R Abilene, R_GÉANT and $\mathrm{R}$ Sprint in Table I each of which contains some transit nodes. For this case, 2DP-NF aims to maximally switch-off all possible links and transit nodes to minimize power usage.

\section{(1) Power Saving}

The power savings of these three topologies are shown in Table II. We see that 2DP-NF can switch off one node and seven links on the Abilene network, with power saving of up to $13.3 \%$; for GÉANT, more than half of transit nodes $(7 / 13)$ can be switched off, and the power saving reaches $39.7 \%$; for Sprint, 7 transit nodes and 87 links can be powered off, achieving $28.5 \%$ power savings.
TABLE II. POWER SAVING WHEN NODES AND LINKS CAN BE SWITCHED-OFF

\begin{tabular}{|c|c|c|c|}
\hline Power Saving & R_Abilene & R_GÉANT & R_Sprint \\
\hline Off Transit Nodes & 1 & 7 & 7 \\
\hline Off Links & 7 & 40 & 87 \\
\hline Power Saving & $13.3 \%$ & $39.7 \%$ & $28.5 \%$ \\
\hline
\end{tabular}

\section{(2) Effects on Link Utilization and Path Length}

Intuitively, switching off nodes and links will affect the link utilization and maximum routing path length (MRPL) since fewer nodes and links are available to carry traffic. Fig. 12, 13 and 14 show the CDF of link utilization and path length for three topologies: R_Abilene, R_GÉANT and R_Sprint with running 2DP-NF and 2DP by shortest path first without powering off nodes and links (2DP-SP).

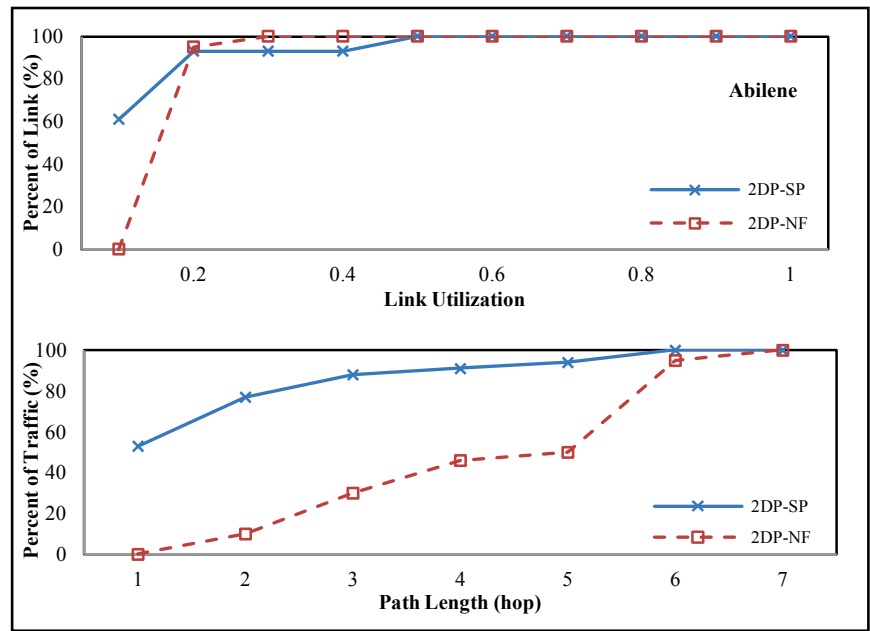

Figure 12. CDF of Link Utilization and Path Length on Abilene

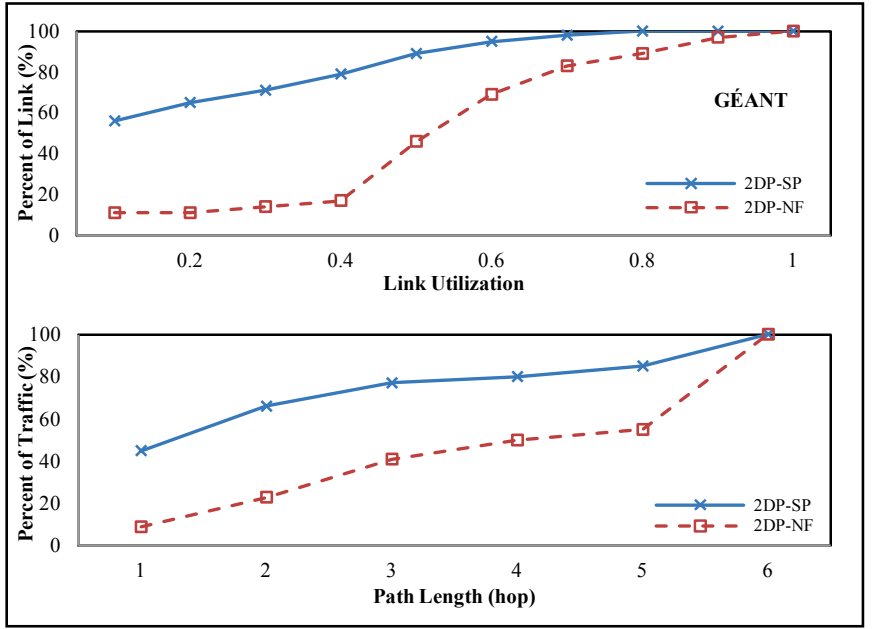

Figure 13. CDF of Link Utilization and Path Length on GÉANT

From Fig. 12 (top), we see that $61 \%$ of links using 2DP-SP have utilization no larger than 0.1 , which is better as compared to $0 \%$ using $2 \mathrm{DP}-\mathrm{NF}$. However, while saving $13.3 \%$ power usage, 2DP-NF also generates $95 \%$ of links with utilization between 0.1 and 0.2 , which is better than 2DP-SP that generates only $32 \%$ of links with utilization in the range. Further, 2DP-NF generates MLU of 0.3, which is better as compared to 2DP-SP with MLU reaching 0.5. For path length, shown in Fig. 12 (bottom), 5\% of traffic demands have MRPL 
(7 hops) larger than the network diameter (6 hops) for 2DP-NF while MRPL of all traffics for 2DP-SP is no larger than network diameter.

In Fig. 13 (top), due to off-peak period, 56\% of links using 2DP-SP have utilization no larger than 0.1 , which is better than the result generated by $2 \mathrm{DP}-\mathrm{NF}$, only at $11 \%$. Different from R_Abilene network, 2DP-SP generates MLU value only at 0.8 on R_GÉANT network, which is better than 2DP-NF, up to 1.0. As shown in Fig. 13 (bottom), for 2DP-SP, 45\% demands route its traffics through single hop paths, but only $9 \%$ demands use single hop routing for 2DP-NF. However, running 2DP-SP and 2DP-NF obtains the same MRPL, which is less than the network diameter, at six hops.

The results for Sprint network are shown in Fig. 14. For link utilization, Fig. 14 (top) shows that 2DP-NF obtains $99 \%$ of links with utilization no larger than 0.8 , which is only $6 \%$ higher than the value generated by 2DP-SP. For routing path length, Fig. 14 (bottom) shows that 2DP-NF increases the length produced by 2DP-SP less as compared to on Abilene and GEANT. In a larger topology, like Sprint, there are more alternative paths for each $\left(s_{d}, t_{d}\right)$ pair that have routing path length closer to the shortest path. Thus 2DP-NF can produce better results in term of path length on Sprint as compared to on the other two networks. Notice that both 2DP-NF and 2DPSP obtain the same MRPL, which is equal to the network diameter (8 hops).

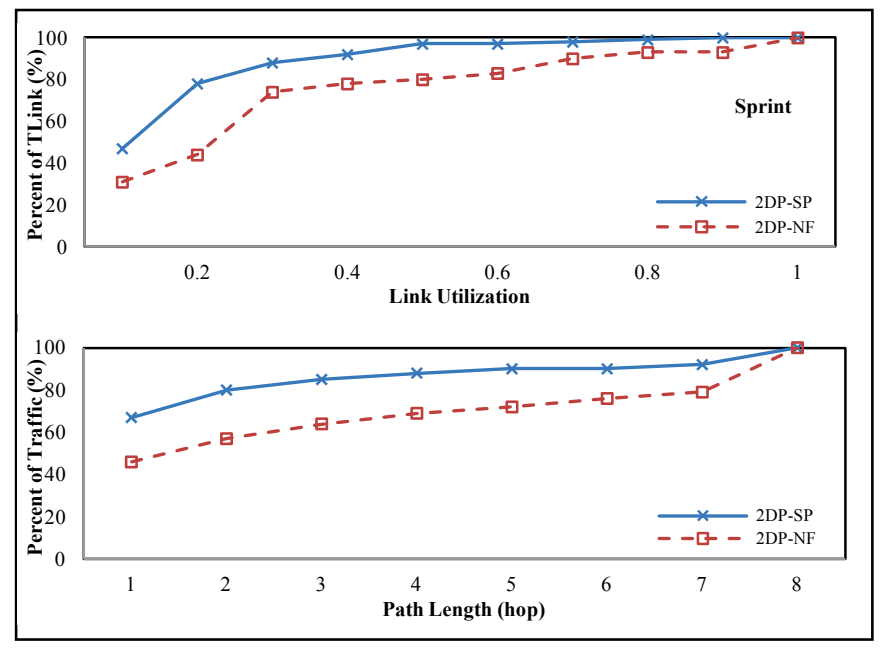

Figure 14. CDF of Link Utilization and Path Length on Sprint

\section{CONClusion}

We have presented a new energy-aware routing problem that aims to maximally switch off unnecessary nodes and links during off-peak periods such that the remaining nodes and links are sufficient to route all traffic demands and that the ratio of using two link-/node-disjoint paths is not less than a given threshold and meets MLU requirement. We have proposed a heuristic technique to solve the problem. Through extensive simulations on both real and synthetic network topologies and traffic demands, we have shown its benefits in reducing the network's energy consumption. In future, we will combine path delay, network reliability and MLU constraints in $2 \mathrm{DP}$ routing for energy-aware traffic engineering.

\section{REFERENCES}

[1] K. Xiong, Z. D. Qiu, Y. Guo, and H. Zhang. "Multi-constrained shortest disjoint paths for reliable QoS routing". In ETRI Journal, vol. 31, no. 5, pp. 534-544, 2009.

[2] Y. Wang, W. Yang, nd J. Wu, "Spare Capacity allocation using partially disjoint paths for dual link failure ". In $D R C N$, Budapest, Hungary, pp. 171-178, 2013.

[3] Y. Guo, F. Kuipers and P. V. Meeghem. "Link-disjoint paths for reliable QoS routing.” In Int. J. Commun. Syst., vol. 26, pp. 779-798, 2003.

[4] K. W. Roth, F. Goldstein and J. Kleinman, "Office and telecommunications equipment in commerical buildings-volume I: Energy consumption baseline”. In Technical Report 7289500, Arthur D. Little, Inc., 2002.

[5] A. Qureshi, "Cutting the electric bill for Internet-scale systems". In $A C M$ SIGCOMM, Barcelona, Spain, pp. 123-134, 2009.

[6] G. Lin, S. Soh, M. Lazarescu and K. Chin, "Power-Aware Routing in Network with Delay and Link Utilization Constraints". In HPSR, Taipei, Taiwan, July, 2013.

[7] R. Tucker, J. Baliga, R. Ayre, K. Hinton, and W. Sorin, "Energy Consumption in IP Networks". In Proc. ECOC Symp. Green ICT, Brussels, Belgium, pp. 1, 2008.

[8] L. Chiaraviglio, M. Mellia, and F. Neri, "Minimizing ISP Network Energy Cost: Formulation and Solutions". In IEEE/ACM Transactions on Networking, vol. 20, pp. 436-476, 2012.

[9] G. Lin, S. Soh, M. Lazarescu and K. Chin, "On the Effects of EnergyAware Traffic Engineering on Routing Reliability". In $A P C C$, Indonesia, August, 2013.

[10] W. Fisher, M. Suchara, and J. Rexford, "Greening backbone networks: reducing energy consumption by shutting off cables in bundled links". In Green Networking, New Delhi, India, pp. 29-34, 2010.

[11] M. Zhang, C. Yi, B. Liu, and B. Zhang, "GreenTE: Power-Aware Traffic Engineering”. In ICNP, Beijing, China, pp. 21-30, 2010.

[12] J. M. Jaffe, "Algorithms for Finding Paths with Multiple Constraints". In Networks, vol. 14, pp. 95-116, 1984.

[13] J. Y. Yen, "Finding the K Shortest Loopless paths in a network". In Management Science, vol. 17, no. 11, 1971.

[14] J. W. Suurballe and R. E. Tarjan. "A quick method for finding shortest pairs of disjoints paths". In Networks, vol. 14, pp. 325-336, 1984.

[15] A. Srinivas and E. Modiano, "Minimum Energy Disjoint Path Routing in Wireless Ad-Hoc Networks". In Proc. ACM/IEEE Int'l Conf. Mobile Computing and Networking (MOBICOM), San Diego, USA, pp. 122133, 2003.

[16] "Yin Zhang's Abilene TM". In http://www.cs.utexas.edu/ yzhang/ research/AbileneTM/.

[17] S. Uhlig, B. Quoitin, J. Lepropre, and S. Balon, "Providing Public Introdomain Traffic Matrices to the Research Community". In $A C M$ SIGCOMM Computer Communication Review, Pisa, Italy, vol. 36, no. 1, pp. 83, 2006.

[18] N. Spring, R. Mahajan, D. Wetherall, and T. Anderson, "Measuring ISP Topologies with Rocketful". In IEEE/ACM Transactions on Networking, vol. 12, no. 1, pp.2-16, 2004.

[19] S. Kandula, D. Katabi, B. Davie, and A. Charny, "Walking the Tightrope: Responsive Yet Stable Traffic Engineering". In $A C M$ SIGCOMM, PA, USA, pp. 253-264, 2005.

[20] "Spirent Signal Delay Network Emulator - DG/OTU/CPRI". In Datasheet of Spirent Communications, Inc., 2010. 